\title{
Design and Modeling of a Snake Robot Based on Worm-Like Locomotion
}

\author{
Alireza Akbarzadeh ${ }^{\text {a,b,* }}$ and Hadi Kalani ${ }^{\mathrm{b}}$ \\ ${ }^{\text {a }}$ Center for Applied Research on Soft Computing and Intelligent Systems (CARSIS), \\ FUM Robotics Research Center, Mashhad, Iran \\ ${ }^{\mathrm{b}}$ Mechanical Engineering Department, Ferdowsi University of Mashhad, Mashhad, Iran \\ Received 21 January 2011; accepted 5 April 2011
}

\begin{abstract}
In this paper we restrict our attention to worm-like, vertical traveling wave locomotion and present detailed kinematics and dynamics of a planar multi-link snake robot. Lagrange's method is used to obtain the robot dynamics. Webots software is used for simulation and to experimentally investigate the effects of link shape on motor torques. Using the dynamics model and Webots simulation, a nine-link snake robot is designed and constructed. Physical experiments are carried out to validate the mathematical model. Webots software is also used to perform simulation and further validate theoretical results. Finally, stability of the snake robot is experimentally investigated.

(C) Koninklijke Brill NV, Leiden and The Robotics Society of Japan, 2012
\end{abstract}

\section{Keywords}

Snake robot, Lagrange method, worm-like locomotion, stability, serpenoid curve

\section{Introduction}

Snake robots offer potential in assisting in areas such as fire-fighting, rescue missions and maintenance due to their high maneuverability and ability to move through tight spaces. These robots are able to bend and adapt to the form of the terrain on which they move. Their cross-section is significantly smaller than their overall length, which enables them to enter small tubes or orifices and get to places inaccessible to other robots. Snakes usually travel in the horizontal plane. On the contrary, worms are thought of to usually move in the vertical plane orthogonal to the supporting plane. The most famous gaits used by snakes are lateral undulatory (serpentine), concertina, sidewinding and rectilinear locomotion [1-19]. Depend-

\footnotetext{
* To whom correspondence should be addressed. E-mail: ali_akbarzadeh_t@yahoo.com 
ing on the environment, snakes may also combine the basic gaits and create new locomotion modes. Non-snake-like gaits do not exist in nature, but are applicable in snake robot locomotion. However, these gaits are less addressed in the literature. The spinning, flapping and traveling wave gaits are some examples of such gaits.

Serpentine locomotion, also known as lateral undulation, is the most frequently used form of snake locomotion used by real snakes. Hirose showed that the key property of snakes in mimicking serpentine locomotion is the difference in the friction coefficients for the tangential and the normal directions with respect to the body [1]. Therefore, the snake-like robot usually needs wheels to realize directional friction. This requirement limits snake robot adaptability to the environment. One of the original studies of snake-like robots was conducted by Hirose [1]. He formulated the serpenoid curve and proved that a snake-like mechanism is able to generate a net forward thrust by applying the appropriate torque along the length of its body. Chen et al. [2] presented a model for traveling wave locomotion, and considered its kinematics and dynamics. They considered the effects of the initial winding angle and friction coefficient on the joint torques during traveling wave locomotion. Chen et al. [3] also showed that one period of traveling wave locomotion can be divided in four phases. These phases are based on the number of joints contacting the ground and the resultant of the friction forces on contacting joints. Saito et al. [4] constructed a snake robot without wheels. This robot has great potential to adapt to various environments at the cost of increased power consumption. They obtained total equations of motion for a multi-link snake robot traveling with serpentine locomotion. Ma et al. [5] also considered formulation of the kinematics and dynamics of three-dimensional (3-D) snake robot and analyzed creeping locomotion. They investigated the motion efficiency of a sinus-lifting motion in comparison with normal creeping locomotion. Dowling [6] presented a novel and practical design for a limbless snake robot and investigated the effects of many materials for use as the ground contact interface and protective sheath. Ma et al. [7] used Newton-Euler equations to derive the dynamic equation for an $n$-link robot creeping on a slope. They considered both symmetrical and unsymmetrical body shapes. They showed that the unsymmetrical body curve increases the robot's performance. Chirikjian and Burdick $[8,9]$ presented a framework for kinematics and motion planning of snake robots. They modeled the snake-inspired robot as a continuous backbone curve, and analyzed the kinematics of gaits that used both 'stationary', similar to inchworm locomotion, and 'traveling' waves, similar to rectilinear snake locomotion. The model does not consider the dynamics of the system.

Hasanzadeh and Akbarzadeh [11] presented a novel gait - forward head serpentine (FHS) - for a two-dimensional (2-D) snake robot. They used a genetic algorithm (GA) to find FHS gait parameters and performed experiments to validate their results. Kalani et al. [12] considered kinematics and dynamics of traveling wave locomotion of a snake robot along a symmetrical and unsymmetrical body curve. They investigated the effects of friction coefficient, initial winding angle and the unsymmetrical factor on the joint torques. They also [13] proposed a novel 
kinematics modeling method for traveling wave gait. Next, they used Webots software for validating their new method. Bayraktaroglu [14] presented an experimental study on a wheel-less snake-like robot with lateral undulation as well as worm-like motion. The mechanical design, trajectory generation and control method are biologically inspired. To derive the dynamics of the snake robots, three modeling techniques - Lagrangian formulation, Newton-Euler formulation and geometric mechanics - are considered, [15, 16]. In his thesis, Transeth [15] presented a novel non-smooth (hybrid) mathematical model for 2-D planar limbless snake robots. Aside from the ground, this model allows a snake robot to push against obstacles. The 2-D model is extended to a non-smooth 3-D mathematical model of a snake robot. He also used the Euler-Lagrange equations and formulated the dynamics of a planar robot in a standard matrix form. Vossoughi et al. [17] presented a novel structure of a snake-like robot. This structure enables passive locomotion in snake-like robots. He used the Gibbs-Appell method to derive dynamic equations for motion in a horizontal plane. Spranklin [20] considered kinematic and dynamic models for snake robots with a rectilinear gait. The mechanism topology changes over the course of the gait. Ma et al. formulated the kinematics and the dynamics of a 2-D snake-like robot in closed form. The robot dynamics were used to analyzed the 2-D creeping locomotion [7, 10, 21-23]. A mathematical model of a snake robot with 2-d.o.f. revolute joints using the Newton-Euler algorithm is presented by Liljebäck et al. [24]. Wang et al. [23] presented a unified dynamic model for locomotion and manipulation of a snake-like robot, and used differential geometry for its analysis. This approach allows comparison between the dynamics of the snake-like robot and those of redundant manipulators. Their approach also provides a novel geometric point of view to better understand the dynamics of snake-like robots. Ye et al. [25] introduced a 3-D snake-like robot called Perambulator-II with high torque, high mobility and fast progression speed. They offered a detailed mechanical design and experimentally demonstrated the serpentine locomotion.

This paper is organized as follows. In Section 2, the curvature function of the serpenoid curve is used to derive the body shape of the robot. In Section 3, the kinematics of the snake robot is developed, and the displacement, velocity and acceleration of the gravity center for each link, as well as that of the snake body, are calculated. In Section 4, the dynamics of the robot using Lagrange's method is obtained. In Section 5, dynamic equations are coded in MATLAB and joint torques are obtained. This section also presents Webots simulation results. In Section 6, the FUM Snake-3 robot design is introduced and the effect of link shape is experimentally evaluated. Furthermore, stability of the robot is also experimentally evaluated. Finally, Section 7 presents some concluding remarks.

\section{Analysis of Worm-Like Locomotion}

The most common type of snake-inspired locomotion is known as the serpentine gait. However, this locomotion requires wheels or treads. However, there exists a 
snake-inspired locomotion - rectilinear motion - that requires pure undulation. There are two forms of rectilinear motions - rectilinear motion that uses vertical waves and rectilinear motion that uses expanding/contracting segments. The second type of locomotion used by the inchworm may be described as creeping motion. In this type of locomotion, parts of the worm body advance forward while other parts of the body are anchored to the terrain. The first type of locomotion - the vertical traveling wave - is also used by both snakes and worms. However, people associate this locomotion mostly with worms and thereby we refer to it as wormlike locomotion.

To make the robot move, we need to change the joint angles in a certain manner. Hirose [1] showed that by changing the joint angles sinusoidally with a common frequency and a phase lag between consecutive joints, the snake locomotion is generated.

The body shape of a snake is described by a series of S-shaped, sinusoidal-like curves that the body forms while in execution. In most robots, this body shape is usually mimicked by utilization of the serpenoid curve, introduced by Hirose [1]. For convenience, we use the serpenoid curve $[2,3,7]$ as the basic body shape of the snake robot traveling with worm-like locomotion. The curvature function of serpenoid curve is defined as:

$$
\rho(s)=\frac{-2 K_{n} \pi \alpha}{L} \sin \left(\frac{2 K_{n} \pi s}{L}\right),
$$

where $L$ is the whole length of snake body, $K_{n}$ is the number of the wave shapes, $\alpha$ is the initial winding angle of the curve and $s$ is the body length along the body curve. See Fig. 1.

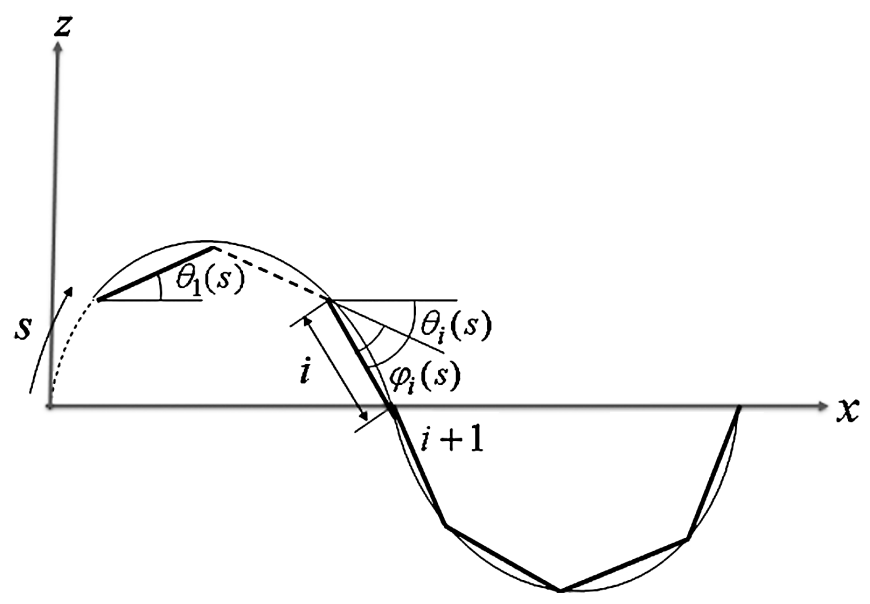

Figure 1. Snake robot along the serpenoid curve. 
Since $\varphi=s \rho$, we can write,

$$
\mathrm{d} \varphi=\rho \mathrm{d} s \rightarrow \varphi=\int_{s+(i-1) l}^{s+i l} \rho(u) \mathrm{d} u=\int_{s+(i-1) l}^{s+i l} \frac{-2 K_{n} \pi \alpha}{L} \sin \left(\frac{2 K_{n} \pi u}{L}\right) \mathrm{d} u .
$$

After simplifying, relative angles are given by,

$$
\varphi_{i}(s)=-2 \alpha \sin \left(\frac{K_{n} \pi}{L}\right) \times \sin \left(\frac{2 K_{n} \pi s}{L}+\frac{2 K_{n} \pi i}{n}-\frac{K_{n} \pi}{n}\right) .
$$

The relative angle velocities and accelerations can be derived by differentiating (3) with respect to time. Then:

$$
\begin{aligned}
\dot{\varphi}_{i}(s)= & \frac{-4 \alpha K_{n} \pi}{L} \sin \left(\frac{K_{n} \pi}{L}\right) \times \sin \left(\frac{2 K_{n} \pi s}{L}+\frac{2 K_{n} \pi i}{n}-\frac{K_{n} \pi}{n}\right) \dot{s} \\
\ddot{\varphi}_{i}(s)= & \frac{-4 \alpha K_{n} \pi}{L} \sin \left(\frac{K_{n} \pi}{L}\right) \times \sin \left(\frac{2 K_{n} \pi s}{L}+\frac{2 K_{n} \pi i}{n}-\frac{K_{n} \pi}{n}\right) \ddot{s} \\
& -\frac{8 \alpha K_{n}^{2} \pi^{2}}{L^{2}} \sin \left(\frac{K_{n} \pi}{L}\right) \times \sin \left(\frac{2 K_{n} \pi s}{L}+\frac{2 K_{n} \pi i}{n}-\frac{K_{n} \pi}{n}\right) \dot{s}^{2} .
\end{aligned}
$$

Using the relation between absolute and relative joint angle values, we obtain:

$$
\theta_{i}=\theta_{1}+\sum_{k=1}^{i-1} \varphi_{k}
$$

where $\theta_{1}$ is the absolute angle for the head link, the first link, and $\theta_{i}$ are absolute angles of subsequent links. The absolute velocities and accelerations can be derived by differentiating (6) with respect to time. Therefore:

$$
\begin{aligned}
& \dot{\theta}_{i}=\dot{\theta}_{1}+\sum_{k=1}^{i-1} \dot{\varphi}_{k} \\
& \ddot{\theta}_{i}=\ddot{\theta}_{1}+\sum_{k=1}^{i-1} \ddot{\varphi}_{k},
\end{aligned}
$$

where $\theta_{i}, \dot{\theta}_{i}$ and $\ddot{\theta}_{i}$ are the absolute value of joint angle, angular velocity and angular acceleration of the $i$ th link with respect to the $x$-axis, respectively.

\section{Kinematics of the Snake Robot}

A planar snake robot consisting of $n$ links, connected through $n-1$, joints is depicted in Fig. 2. Each link is rigid with uniformly distributed mass and is equipped with a torque actuator (motor). Each link is of mass $m_{i}$, length $l_{i}$ and moment of inertia $I_{i}$. Let $\left(x_{\mathrm{c} i}, z_{\mathrm{c} i}\right)$ and $\theta_{i}$ define the center of gravity and the angle between 


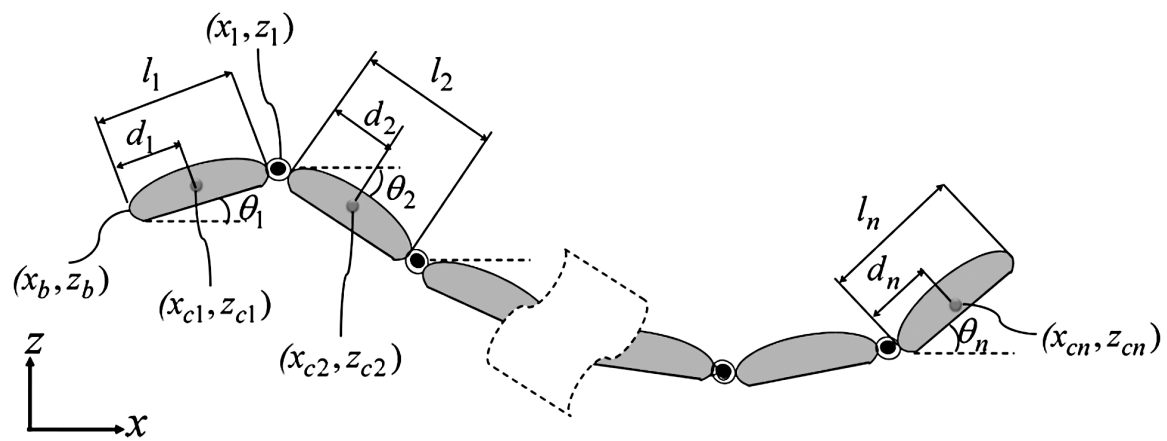

Figure 2. $n$-link snake robot.

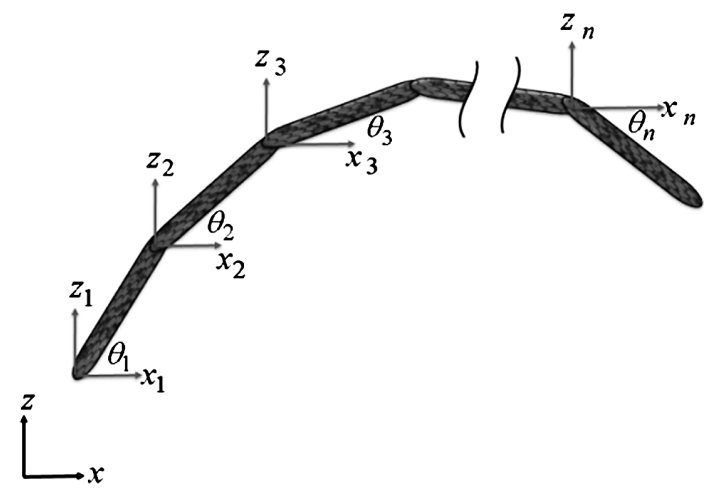

Figure 3. Coordinate frames for each link.

the link and the $x$-axis, respectively. Values of $d_{i}$ represent the location of the mass center of the $i$ th link. $\left(x_{\mathrm{b}}, z_{\mathrm{b}}\right)$ is the coordinate of the head link.

To define a kinematics model for the robot, we attach a coordinate system to each link of the robot. See Fig. 3. The linear position, velocity and acceleration of the $i$ th joint can be written as:

$$
\begin{gathered}
x_{i}=x_{\mathrm{b}}+\sum_{j=1}^{i-1} l_{j} \cos \theta_{j} \\
z_{i}=z_{\mathrm{b}}+\sum_{j=1}^{i-1} l_{j} \sin \theta_{j} \\
\dot{x}_{i}=\dot{x}_{\mathrm{b}}-\sum_{j=1}^{i-1} l_{j} \dot{\theta}_{j} \sin \theta_{j} \\
\dot{z}_{i}=\dot{z}_{\mathrm{b}}+\sum_{j=1}^{i-1} l_{j} \dot{\theta}_{j} \cos \theta_{j}
\end{gathered}
$$




$$
\begin{aligned}
& \ddot{x}_{i}=\ddot{x}_{\mathrm{b}}-\sum_{j=1}^{i-1} l_{j} \ddot{\theta}_{j} \sin \theta_{j}-\sum_{j=1}^{i-1} l_{j} \dot{\theta}_{j}^{2} \cos \theta_{j} \\
& \ddot{z}_{i}=\ddot{z}_{\mathrm{b}}+\sum_{j=1}^{i-1} l_{j} \ddot{\theta}_{j} \cos \theta_{j}-\sum_{j=1}^{i-1} l_{j} \dot{\theta}_{j}^{2} \sin \theta_{j},
\end{aligned}
$$

where $i=1,2, \ldots, n$. Similarly, the position, velocity and acceleration of the gravity centers of the $i$ th link can be obtained as:

$$
\begin{aligned}
& x_{\mathrm{c} i}=x_{\mathrm{b}}+\sum_{j=1}^{i-1} l_{j} \cos \theta_{j}+d_{i} \cos \theta_{i} \\
& z_{\mathrm{c} i}=z_{\mathrm{b}}+\sum_{j=1}^{i-1} l_{j} \sin \theta_{j}+d_{i} \sin \theta_{i} \\
& \dot{x}_{\mathrm{c} i}=\dot{x}_{\mathrm{b}}-\sum_{j=1}^{i-1} l_{j} \dot{\theta}_{j} \sin \theta_{j}-d_{i} \dot{\theta}_{i} \sin \theta_{i} \\
& \dot{z}_{\mathrm{c} i}=\dot{z}_{\mathrm{b}}+\sum_{j=1}^{i-1} l_{j} \dot{\theta}_{j} \cos \theta_{j}+d_{i} \dot{\theta}_{i} \cos \theta_{i} \\
& \ddot{x}_{\mathrm{c} i}=\ddot{x}_{\mathrm{b}}-\sum_{j=1}^{i-1} l_{j} \dot{\theta}_{j} \ddot{\theta}_{j} \sin \theta_{j}-\sum_{j=1}^{i-1} l_{j} \dot{\theta}_{j}^{2} \cos \theta_{j}-d_{i} \ddot{\theta}_{i} \sin \theta_{i}-d_{i} \theta_{i}^{2} \cos \theta_{i} \\
& \ddot{z}_{\mathrm{c} i}=\ddot{z}_{\mathrm{b}}+\sum_{j=1}^{i-1} l_{j} \dot{\theta}_{j} \ddot{\theta}_{j} \cos \theta_{j}-\sum_{j=1}^{i-1} l_{j} \dot{\theta}_{j}^{2} \sin \theta_{j}+d_{i} \ddot{\theta}_{i} \cos \theta_{i}+d_{i} \theta_{i}^{2} \sin \theta_{i} .
\end{aligned}
$$

The snake robot is constructed with $n$ links and, therefore, the gravity center of the entire snake robot's body is obtained as:

$$
M X_{\mathrm{C}}=\sum_{i}^{n} m x_{\mathrm{c} i}, \quad M Z_{\mathrm{C}}=\sum_{i}^{n} m z_{\mathrm{c} i},
$$

where $M$ and $\left(X_{\mathrm{C}}, Z_{\mathrm{C}}\right)$ represent the total mass and position of the mass center for the snake robot, respectively. The displacement, velocity and acceleration of the gravity center of the snake body are obtained by:

$$
\begin{aligned}
& X_{\mathrm{C}}=\frac{\sum_{i=1}^{n} m x_{\mathrm{c} i}}{M}=\frac{1}{n} \sum_{i=1}^{n} x_{i} \\
& \dot{X}_{\mathrm{C}}=\frac{\sum_{i=1}^{n} m \dot{x}_{\mathrm{c} i}}{M}=\frac{1}{n} \sum_{i=1}^{n} \dot{x}_{i}
\end{aligned}
$$




$$
\begin{gathered}
\ddot{X}_{\mathrm{C}}=\frac{\sum_{i=1}^{n} m \ddot{x}_{\mathrm{c} i}}{M}=\frac{1}{n} \sum_{i=1}^{n} \ddot{x}_{i} \\
Z_{\mathrm{C}}=\frac{\sum_{i=1}^{n} m z_{\mathrm{c} i}}{M}=\frac{1}{n} \sum_{i=1}^{n} z_{i} \\
\dot{Z}_{\mathrm{C}}=\frac{\sum_{i=1}^{n} m \dot{z}_{\mathrm{c} i}}{M}=\frac{1}{n} \sum_{i=1}^{n} \dot{z}_{i} \\
\ddot{Z}_{\mathrm{C}}=\frac{\sum_{i=1}^{n} m \ddot{z}_{\mathrm{c} i}}{M}=\frac{1}{n} \sum_{i=1}^{n} \ddot{z}_{i},
\end{gathered}
$$

where $X_{\mathrm{C}}$ and $Z_{\mathrm{C}}, \dot{X}_{\mathrm{C}}$ and $\dot{Z}_{\mathrm{C}}$, and $\ddot{X}_{\mathrm{C}}$ and $\ddot{Z}_{\mathrm{C}}$ represent the displacement, velocity and acceleration of the gravity center of the snake body, respectively.

\section{Dynamics Modeling}

The formulation of dynamics of the snake robot traveling in worm-like locomotion is presented in this section. A dynamics model is necessary in order to determine the amount of torque required to actuate the robot. Furthermore, a dynamics model can be used in simulation, which better enables performance optimization. One of the challenges of the snake robot is deriving its dynamic equation and generalizing it to a snake robot with $n$ links. In the present paper, Lagrange's method is used and the dynamics model of the robot is obtained.

\subsection{Interaction of the Robot with the Enviroment}

A snapshot of a snake robot traveling in a wave locomotion mode is shown in Fig. 4. During worm-like locomotion, as links travel through the body curve, there are instances when the full length of each link comes in contact with the ground. This time is assumed to be significantly low and, thus, negligible. Further, in this paper, we assume $K_{n}=2$ and, therefore, there are at least two contact points with the ground and subsequently at least two friction forces. To generate locomotion, the

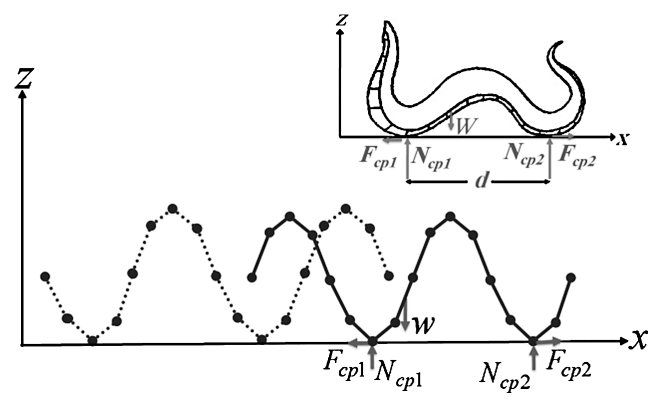

Figure 4. Actual worm and its schematic equivalent external forces on the worm body. 
resultant of these two friction forces should be high enough to transmit the traveling wave. The velocity of this locomotion dependent on the transmitting velocity of the wave along the body. The values of friction forces dependent on the body shape, which subsequently decides the amount of pressure on the supporting plane. To model the friction forces, we consider a simple Coulomb friction model as:

$$
F_{i}=-\mu \cdot \operatorname{sign}(v) \cdot N_{i},
$$

where $\mu$ is the friction coefficient between the contacting joint and the supporting plane. The signum function is denoted by $\operatorname{sign}(v)$. In other words, the value of this function will be 1 if $v>0,0$ if $v=0$ and -1 if $v<0$. Further, neglecting the state where the links are in the horizontal state allows simplification of the dynamic analysis. It should be noted that while in motion, both contact points are in motion and never stationary. However, one contact point is relatively static with respect to the supporting plane, while the other contact point slides on the supporting plane. The resultant force is the difference of the two friction forces, which should be high enough to meet the need for transmitting the traveling wave to generate locomotion.

Consider Fig. 4. Newton's second principle is applied to the free-body diagram of the snake robot. Therefore:

$$
\begin{gathered}
N_{\mathrm{cp} 2}=\frac{1}{d}\left[\left(W+M \ddot{Z}_{\mathrm{C}}\right)\left(x_{\mathrm{c}}-x_{\mathrm{cp} 1}\right)-M \ddot{X}_{\mathrm{C} z_{\mathrm{c}}}\right] \\
N_{\mathrm{cp} 2}-W+N_{\mathrm{cp} 1}=M \ddot{Z}_{\mathrm{C}},
\end{gathered}
$$

where $d$ is the distance between the two supporting points, $W$ is overall weight of the snake, $N$ represents the supporting force, $M \ddot{X}_{\mathrm{C}}$ and $M \ddot{Z}_{\mathrm{C}}$ are the inertial forces of the snake robot body along the $X$ - and $Z$-axis, respectively. Finally, cp1 and cp2 represents the two contact points. Equations (28)-(30) can be used to determine the values of the two supporting forces, $N_{\mathrm{c} 1}$ and $N_{\mathrm{c} 2}$, as well as the driving force, which is the difference between the two frictional forces.

\subsection{Lagrange Method}

The instantaneous system configuration will be known upon having $\left(x_{\mathrm{b}}, z_{\mathrm{b}}\right)$ and $\theta_{i}$ $(1 \leqslant i \leqslant n)$. Therefore, the generalized coordinates are selected as:

$$
q=\left[\theta_{1}, \theta_{2}, \theta_{3}, \ldots, \theta_{n}, x_{\mathrm{b}}, z_{\mathrm{b}}\right] .
$$

The equations of motion can be written as,

$$
\frac{\mathrm{d}}{\mathrm{d} t}\left(\frac{\partial T}{\partial q_{i}}\right)-\frac{\partial K}{\partial q_{i}}+\frac{\partial V}{\partial q_{i}}=Q_{i}^{\mathrm{nc}} \quad(i=1,2,3, \ldots, n+2),
$$

where $K$ is kinetic energy and $V$ is potential energy. Non-conservative forces, $Q_{i}^{\text {nc }}$, that do work when generalized coordinates are given virtual displacements are actuators torques, friction forces and supporting forces. Therefore, generalized forces may be defined as:

$$
Q_{\theta j}=-l_{j}\left[\sin \theta_{j} \sum_{i=j+1}^{n} f_{x i}\right]+l_{j}\left[\cos \theta_{j} \sum_{i=j+1}^{n} N_{z i}\right]+\tau_{j-1}-\tau_{j}
$$




$$
\begin{gathered}
Q_{x \mathrm{~b}}=\sum_{i=j+1}^{n} f_{x i} \\
Q_{z \mathrm{~b}}=\sum_{i=j+1}^{n} N_{z i},
\end{gathered}
$$

where $f_{x i}$ is friction force, $N_{z i}$ is supporting force and $Q_{\theta j}$ are generalized forces related to the generalized coordinate $\theta_{j} . Q_{x \mathrm{~b}}$ and $Q_{z \mathrm{~b}}$ are generalized forces related to $x_{\mathrm{b}}$ and $z_{\mathrm{b}}$, respectively. The kinetic energy of the $n$-link snake robot can be defined as:

$$
K=\sum_{i=1}^{n}\left[\frac{1}{2} I_{i} \dot{\theta}_{i}^{2}+\frac{1}{2} m_{i}\left(\dot{x}_{i}^{2}+\dot{z}_{i}^{2}\right)\right] .
$$

Substituting (17) and (18) into (36), we conclude:

$$
\begin{aligned}
K= & \sum_{i=1}^{n}\left[\frac{1}{2}\left(I_{i}+m_{i} d_{i}^{2}\right) \dot{\theta}_{i}^{2}+\frac{1}{2} m_{i}\left(\dot{x}_{i}^{2}+\dot{z}_{i}^{2}\right)\right] \\
& +\left\{\sum_{i=1}^{n} m_{i} d_{i} \dot{\theta}_{i} \sum_{j=1}^{i-1}\left[l_{j} \theta_{j} \cos \left(\theta_{i}-\theta_{j}\right)\right]\right\} \\
& +\sum_{i=1}^{n}\left[m_{i} d_{i} \dot{\theta}_{i}\left(\dot{z}_{\mathrm{b}} \cos \theta_{i}-\dot{x}_{\mathrm{b}} \sin \theta_{i}\right)\right] \\
& +\sum_{i=1}^{n}\left\{m_{i} \sum_{j=1}^{i-1}\left[l_{j} \dot{\theta}_{j}\left(\dot{z}_{\mathrm{b}} \cos \theta_{j}-\dot{x}_{\mathrm{b}} \sin \theta_{j}\right)\right]\right\} \\
& +\sum_{i=1}^{n}\left\{\frac{1}{2} m_{i}\left(\sum_{j=1}^{i-1}\left[l_{j} \dot{\theta}_{j} \sin \theta_{j}\right]\right)^{2}+\frac{1}{2} m_{i}\left(\sum_{j=1}^{i-1}\left[l_{j} \dot{\theta}_{j} \cos \theta_{j}\right]\right)^{2}\right\}
\end{aligned}
$$

Next, consider potential energy defined as:

$$
V=\sum_{i=1}^{n} m_{i} g z_{\mathrm{c} i}
$$

By replacing (16) into (38), we have:

$$
V=\sum_{i=1}^{n} m_{i} g z_{\mathrm{c} i}=\sum_{i=1}^{n}\left\{m_{i} g\left[z_{\mathrm{b}}+\sum_{j=1}^{i-1} l_{j} \sin \theta_{j}+d_{i} \sin \theta_{i}\right]\right\} .
$$

Equations (33)-(39) can be placed into the Lagrangian formulation, (32) and the dynamic model for the $n$-link snake robot can be derived as:

$$
\mathbf{B T}=\mathbf{M}(\theta) \ddot{\mathbf{q}}+\mathbf{H}(\theta, \dot{\theta})+\mathbf{F}(\theta)+\mathbf{G}(\theta)+\mathbf{S}(\theta),
$$


where $\mathbf{M}_{(n+2) \times(n+2)}$ is a positive definite and symmetric inertia matrix, $\mathbf{H}_{(n+2) \times 1}$ matrix represents centrifugal and Coriolis terms, $\mathbf{F}_{(n+2) \times 1}$ matrix represents friction forces, $\mathbf{B}_{(n+2) \times(n-1)}$ is a constant matrix, $\mathbf{G}_{(n+2) \times 1}$ matrix represents the potential energy, $\mathbf{S}_{(n+2) \times 1}$ matrix represents supporting forces, $\mathbf{T}_{(n-1) \times 1}$ matrix represents input torques, and $\mathbf{q}, \dot{\mathbf{q}}, \ddot{\mathbf{q}}$ are the $(n+2) \times 1$ matrix of generalized coordinates and their derivatives. Finally, $\boldsymbol{\theta}, \dot{\boldsymbol{\theta}}, \ddot{\boldsymbol{\theta}}$ are the $n \times 1$ matrix of links absolute angles and their derivatives. The detailed forms of $\mathbf{M}, \mathbf{H}, \mathbf{B}, \mathbf{G}, \mathbf{F}$ and $\mathbf{S}$ in (40) are presented in the Appendix.

\subsubsection{Forward Dynamics}

In forward dynamics, input joint torques are supplied and the motion of the snake robot is determined. To do this, (40) is solved using the Euler method. Equation (40) is a $(n+2)$-dimensional linear equation of $(n+2)$ unknown variables $\left(\ddot{q} \in R^{n+2}\right)$. Upon solving this equation, angular acceleration for all links $\left(\ddot{\theta} \in R^{n}\right)$ as well as the acceleration of a point at the head link $\left(\ddot{x}_{\mathrm{b}}, \ddot{z}_{\mathrm{b}}\right)$ can be obtained. Next, angular position and velocities of the first link, as well as linear position and velocity, $\left(x_{\mathrm{b}}, z_{\mathrm{b}}, \dot{x}_{\mathrm{b}}, \dot{z}_{\mathrm{b}}\right)$, of a point at the head link, can all be obtained through integration. Therefore, snake robot motion is derived for when input torques for all joints are supplied.

\subsubsection{Inverse Dynamics}

In inverse dynamics, desired time histories of relative angles of the adjacent links are supplied and required motor torques are obtained. In other words, given instantaneous relative angles and their derivatives $(\varphi, \dot{\varphi}, \ddot{\varphi})$, along with initial conditions, (40) can be solved in order to find the required torques and coordinates of the head of the robot. The relation between absolute and relative values of joint angles is:

$$
\varphi_{i}=\theta_{i+1}-\theta_{i}, \quad i=1,2, \ldots, n-1 .
$$

Equation (41) can be written in matrix form as:

$$
\theta=E \varphi+e \theta_{1},
$$

where $\varphi$ is an $n$-dimensional vector of $\left[\varphi_{1}, \varphi_{2}, \ldots, \varphi_{n-1}\right], \theta_{1}$ is the absolute angle of the head, $E_{i j}$ and $e$ are defined as:

$$
e=[1,1, \ldots, 1]^{\mathrm{T}} \quad \text { and } \quad E_{i j}= \begin{cases}1 & i>j \\ 0 & \text { others. }\end{cases}
$$

The dynamic equation (40) is decoupled into two parts:

$$
\begin{gathered}
{ }^{p} M(\theta) \ddot{\theta}+{ }^{p} N(\theta) \ddot{r}_{\mathrm{b}}+{ }^{p} H(\theta, \dot{\theta})+{ }^{p} f(\theta)+{ }^{p} G(\theta)+{ }^{p} S(\theta)=D \tau \\
{ }^{q} M(\theta) \ddot{\theta}+{ }^{q} N(\theta) \ddot{r}_{\mathrm{b}}+{ }^{p} H(\theta, \dot{\theta})+{ }^{q} f(\theta)+{ }^{q} G(\theta)+{ }^{q} S(\theta)=0,
\end{gathered}
$$

where:

$$
M=\left[\begin{array}{cc}
{ }^{p} M_{n \times n} & { }^{p} N_{n \times 2} \\
{ }^{q} M_{2 \times n} & { }^{q} N_{2 \times 2}
\end{array}\right], \quad H=\left[\begin{array}{c}
{ }^{p} H_{n \times 1} \\
{ }^{q} H_{2 \times 1}
\end{array}\right], \quad r_{\mathrm{b}}=\left[\begin{array}{c}
x_{\mathrm{b}} \\
z_{\mathrm{b}}
\end{array}\right] .
$$


By substituting the second derivative of (42) into (45), we obtain:

$$
\begin{aligned}
\ddot{r}_{\mathrm{b}} & =-{ }^{q} N^{-1}\left({ }^{q} M \ddot{\theta}+{ }^{q} H+{ }^{q} f+{ }^{q} G+{ }^{q} S\right) \\
& =-{ }^{q} N^{-1 q} M\left(E \ddot{\varphi}+e \ddot{\theta}_{1}\right)-{ }^{q} N^{-1}\left({ }^{q} H+{ }^{q} f+{ }^{q} G+{ }^{q} S\right) .
\end{aligned}
$$

Substituting (47) into (44), we have:

$$
\begin{aligned}
D \tau+ & \left({ }^{p} N^{q} N^{-1 q} M-{ }^{p} M\right) e \ddot{\theta}_{1} \\
= & \left({ }^{p} M-{ }^{p} N^{q} N^{-1 q} M\right) E \ddot{\varphi}-{ }^{p} N^{q} N^{-1}\left({ }^{q} H+{ }^{q} f+{ }^{q} G+{ }^{q} S\right) \\
& +{ }^{p} H+{ }^{p} f+{ }^{p} G+{ }^{p} S .
\end{aligned}
$$

Equation (48) is an $n$-dimensional linear equation of $n$ unknown variables $\ddot{\theta}_{1} \in R$ and $\tau \in R^{n-1}$. Finally, by solving (48), the joint torques, $\tau_{i}$, and the head link angular acceleration, $\ddot{\theta}_{1}$, can be obtained. Substituting these values back into (47), acceleration of the head link, $\ddot{r}_{\mathrm{b}}$, will be obtained. Subsequently, the head link angular velocity and position $\left(\dot{\theta}_{1}, \theta_{1}\right)$ as well as head link linear velocity and position $\left(\dot{r}_{\mathrm{b}}, r_{\mathrm{b}}\right)$, can all be obtained through integration. The complete parameters defining robot motion are now derived for the case when changes in body shape are known. Therefore, upon specifying changes in body shape, the necessary joint torques to generate the desired robot motion can be obtained.

\section{Computer Simulation}

In this section, worm-like locomotion is simulated. The robot model used in the simulation is assumed to have 16 links and 15 joints. Each joint has 1 rotational d.o.f. around axis $\hat{\mathbf{e}}_{y i}$ with angle $\boldsymbol{\theta}_{y i}$. Each link is estimated by a uniform slender rod. To define the body shape, serpenoid curve parameters are used. Table 1 shows the parameters used in the simulation. by:

The body curve is changed with regard to $s, \dot{s}$ and $\ddot{s}$. The acceleration $\ddot{s}$ is given

$$
\ddot{s}= \begin{cases}a & 0 \leqslant t<T / 10 \\ 0 & T / \leqslant t<9 T / 10 \\ -a & 9 T / 10 \leqslant t<T,\end{cases}
$$

Table 1.

Simulation conditions

\begin{tabular}{llll}
\hline Robot parameters & & Serpenoid curve parameters \\
\hline Link length, $l(\mathrm{~m})$ & 0.1 & initial winding angle $(\mathrm{rad})$ & $\pi / 6$ \\
Number of links, $n$ & 16 & total length of snake $(\mathrm{m})$ & 1.6 \\
Mass of a each link, $m(\mathrm{~kg})$ & 0.1 & number of S-shape, $K_{n}$ & 2.0 \\
Coefficient of dynamic friction, $\mu$ & 0.3 & & \\
\hline
\end{tabular}




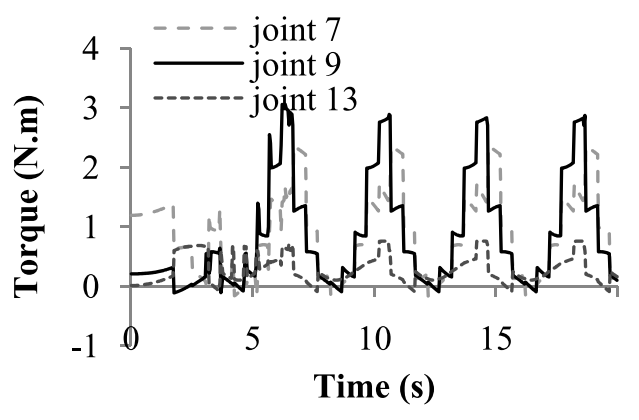

Figure 5. Joint torques when $\alpha=\pi / 6$ and $\mu=0.3$.

where $T$ is the simulation time, assumed to be $20 \mathrm{~s}$, and $a=0.0625 \mathrm{~m} / \mathrm{s}^{2}$. Further, the initial winding angle is assumed to be $\theta_{1}=\alpha$. The initial position and velocity of a point on the tip of the head link are selected as $x_{1}=z_{1}=0$ and $\dot{x}_{1}=\dot{z}_{1}=\dot{\theta}_{1}=0$, respectively. MATLAB software is used for simulation. Dynamic equation (40) is used, and outputs such as joint angles, joint torques and trace of the head link with respect to time are determined.

\subsection{Joint Torques}

The values of joint torques for joints 7,9 and 13 during simulation time are shown in Fig. 5. As can be seen, joint torques are periodic, and amount of maximum joint torques for joints 7 and 9 are in increasing order except joint 13, which shows lower torque. This is because joint 9 is the nearest joint to the gravity center of the snake robot. In other words, as joints get closer to the gravity center, the required maximum torque increases. This finding is similar to what is reported in the earlier literature [2] and the authors' earlier work [12].

\subsection{Simulation of the Snake Robot in Webots ${ }^{\mathrm{TM}}$ Software}

Webots software is used for simulation. Webots is a popular commercial software used for mobile robotics simulation, and provides a rapid prototyping environment for modeling, programming and simulation. The same simulation conditions as before are used except for the difference in definition of the link shape. For the theoretical method — the Lagrangian method — snake links were assumed to be slender rods. In Webots, the links are assumed to be rather a rectangular shape with the leading side having a round edge. A snapshot of the 16-link snake robot is shown in Fig. 6.

Joint 9 is selected for the comparison as this joint experiences the maximum amount of joint torque. The torque values for joint 9 obtained from Webots simulation and the Lagrangian method are both shown in Fig. 7.

As can be seen, both results have a similar magnitude and similar frequency. Therefore, one may conclude that the established kinematics and dynamics of the snake robot are reasonable. 


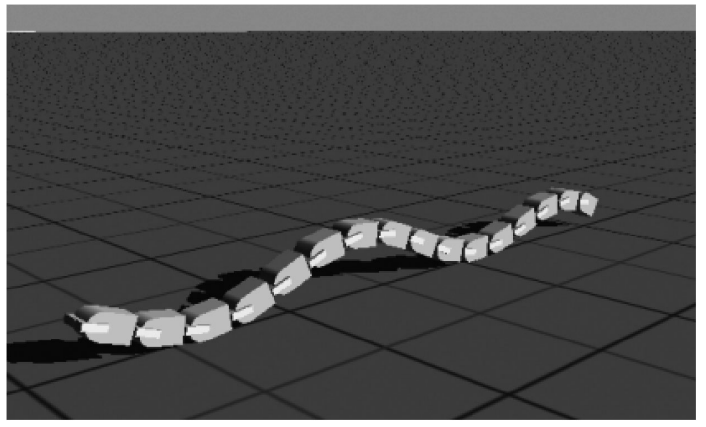

Figure 6. Simulation of the snake robot in Webots.

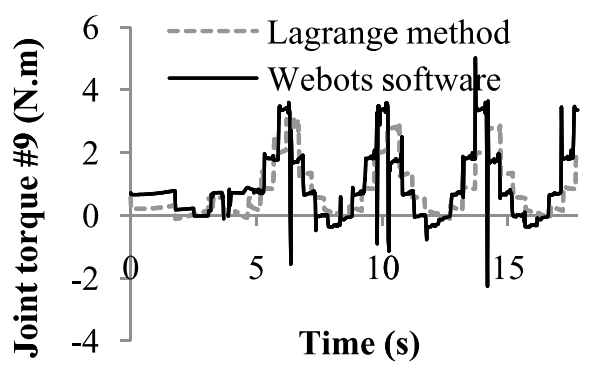

Figure 7. Comparison of Webots and the Lagrangian method for the torque of joint 9.

\section{FUM Snake-3 Robot Design}

FUM Snake-3, shown in Fig. 8, is the third-generation snake robot designed in the FUM Robotics Research Center. The first two robots were designed for serpentine locomotion. FUM Snake-3 is designed to move in the vertical plane. It is made of nine links. Each link is made of two curvilinear shaped Plexiglas sidewalls with 0.25 inch thickness. All nine links have equal length and are designed to avoid collision between the two links as they rotate. This design allows a maximum angle of $125^{\circ}$ rotation between any two adjacent links. The robot in its fully flat configuration has width $75 \mathrm{~mm}$, height $60 \mathrm{~mm}$ and length $670 \mathrm{~mm}$. The overall weight of the snake robot, including all motors and all other components is $1.4 \mathrm{~kg}$. FUM Snake-3 is designed to be able to lift its head or some sections of its body. This will enable the head link or other parts of the snake to cross a gap. This is accomplished by using Dynamixel-AX12 servomotors that could provide a maximum of $1.5 \mathrm{Nm}$ torque. The maximum joint speed is $360^{\circ} \mathrm{s}$. The designed modules are shown in Fig. 9.

The control components are shown in Fig. 10. The locomotion algorithms are implemented in a micro-controller, with the Windows Embedded CE 6.0 operating system. The positions of each servo are sent by RS232 serial communication to the micro controller Friendly ARM board, ARM9 (Mini 2440). The ARM9 is connected by means of a cable to the motors and generates the pulse-width modulated signals for positioning the servos. Distributed control is used. Each motor is 


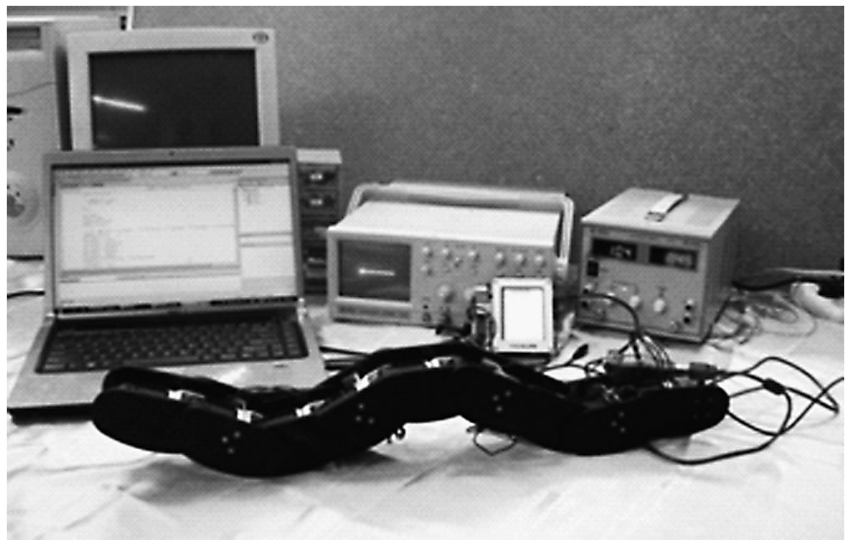

Figure 8. FUM Snake-3.
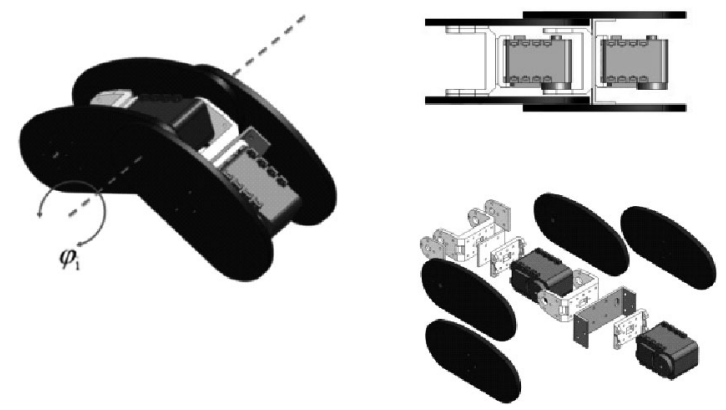

Figure 9. Two link modules assembled and exploded.

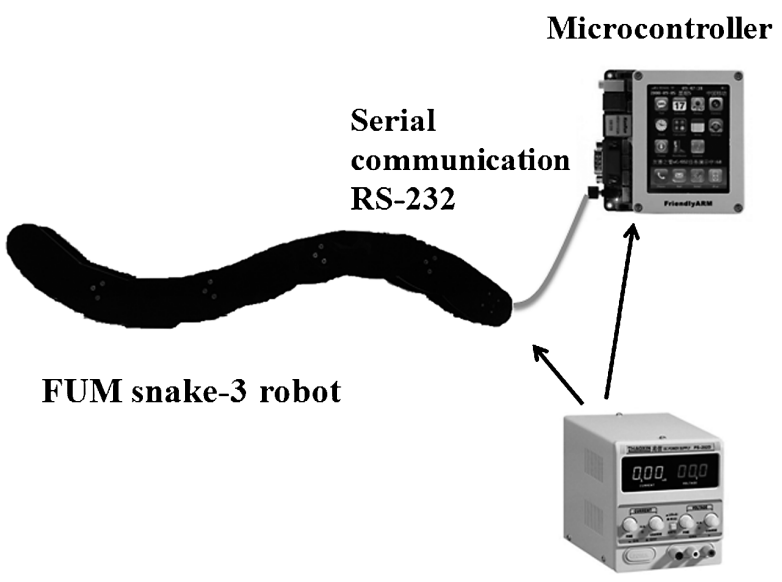

Power supply

Figure 10. Control scheme of the modular robot. 
equipped with a PD controller. The position and speed is controlled with a resolution of 1024 steps.

\subsection{Link Shape}

A critical parameter affecting the quality of the locomotion is shape of the Plexiglas sidewalls. This shape ultimately determines the link shape for the robot. Therefore, once the robot was designed in Solidworks, it was quickly simulated in Webots software. The Plexiglas sidewalls were initially designed to have a rectangular shape. Obtaining as smooth motion as possible is the goal. However, the simulation of the rectangular-shaped Plexiglas links showed the existence of undesirable impact forces as the result of sharp edges of the rectangular Plexiglas hitting the ground. We propose changing the shape of this edge to curvilinear, which results in a significant lowering of the impact forces experienced by the robot. Therefore, a novel shape for the Plexiglas sidewalls is utilized. Webots software was used as simulation. As expected, the impact continued to decrease as we got away from the sharp edge. The final shape of the Plexiglas sidewalls is shown in Fig. 8. The effect of this shape and the original flat link on the torque of joint 9 are shown in Fig. 11. As Fig. 11 demonstrates, the undesirable high peaks are significantly reduced.

The progression of the snake robot and its corresponding Webots simulation is shown in Fig. 12.

Hopkins [19] investigated the relationship between snake-inspired robot dimensions, performance and velocity. He found that snake robots share many common characteristics that allow them to be easily grouped under a general classification (Table 2).

Similar to Ref. [19], the performance of the FUM Snake-3 robot is graphically compared with the other robot designs as given in Table 2 . The maximum velocity versus (a) total robot length (b) robot cross-section and (c) total robot weight are plotted and shown in Fig. 13.

From Table 2, we note that snake robots using rectilinear-based locomotion are generally slower than the other robots. Considering that FUM Snake-3 uses worm-

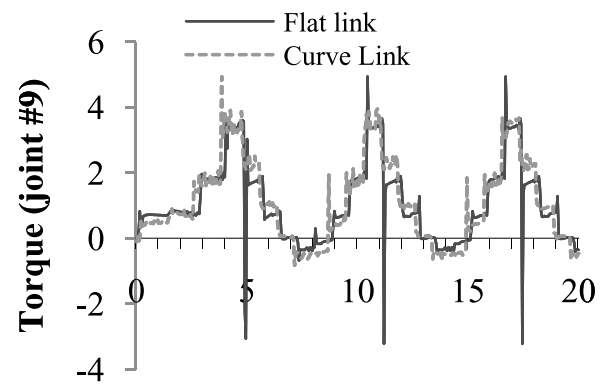

Time (s)

Figure 11. The effect of this shape and the original flat link on the torque of joint 9. 


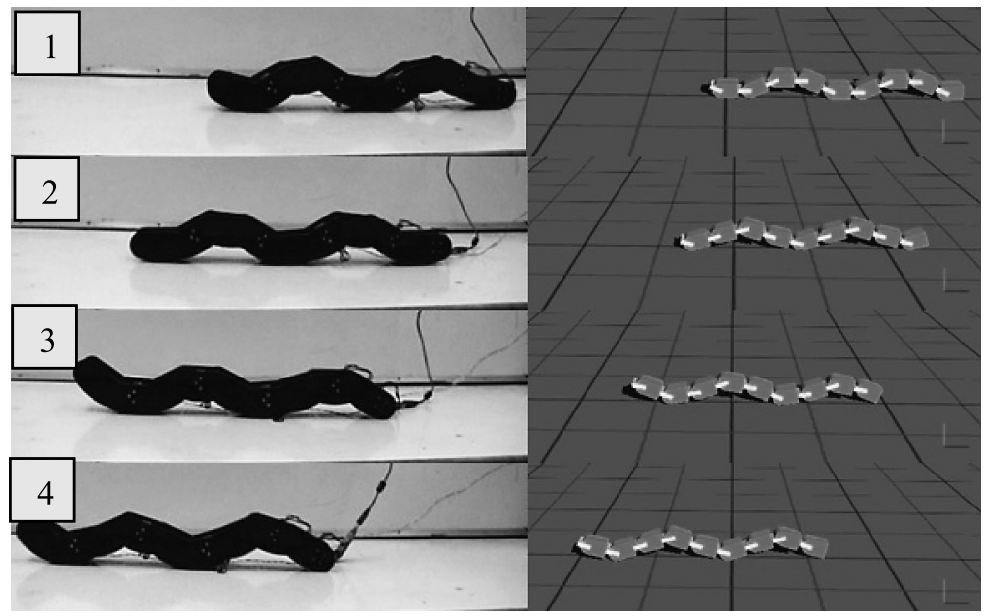

Figure 12. Progression of the snake robot in real and virtual environments.

\section{Table 2.}

Snake-inspired robot dimensions and performance [19]

\begin{tabular}{llcllcc}
\hline Robot & Category & $\begin{array}{l}\text { Overall } \\
\text { length } \\
(\mathrm{mm})\end{array}$ & $\begin{array}{l}\text { Cross- } \\
\text { section } \\
\left(\mathrm{m}^{2}\right)\end{array}$ & $\begin{array}{l}\text { Overall } \\
\text { weight } \\
(\mathrm{kg})\end{array}$ & $\begin{array}{l}\text { Velocity } \\
(\mathrm{mm} / \mathrm{s})\end{array}$ & $\begin{array}{l}\text { Number of } \\
\text { links or } \\
\text { modules }^{\mathrm{a}}\end{array}$ \\
\hline ACM III & I & 2000 & 0.023 & 28 & 400 & 20 \\
AmphiBot I (AB I) & I & 490 & 0.002 & - & 35 & 8 \\
AmphiBot II (AB II) & I & 770 & 0.002 & - & 400 & 8 \\
KR-II & II & 3300 & 0.497 & 370 & 500 & 7 \\
KR-I & III & 1390 & 0.081 & 27.8 & 266 & 6 \\
OmniTread (OT-8) & III & 1270 & 0.034 & 13.6 & 100 & 5 \\
OmniTread (OT-4) & III & 940 & 0.007 & 3.6 & 150 & 7 \\
JL-I & III & 1050 & 0.038 & 21 & 180 & 3 \\
Kotay's Inchworm I (KIR-1) & IV & 250 & - & 0.455 & 4 & - \\
Kotay's Inchworm II (KIR-2) & IV & 330 & - & 0.566 & 13 & - \\
CMU (M1) & IV & 840 & 0.003 & 1.26 & 102 & - \\
FUM Snake-3 & IV & $\mathbf{6 7 0}$ & $\mathbf{0 . 0 0 4 5}$ & $\mathbf{1 . 4}$ & $\mathbf{1 0 5}$ & $\mathbf{9}$ \\
Planar Inchworm (PI) & V & 710 & - & 6 & 1 & 2 \\
Slim Slime Robot (SSR) & V & 730 & 0.013 & 12 & 60 & 6 \\
\hline
\end{tabular}

a New entry to original table.

I, robots with passive wheels; II, robots with active wheels; III, robots with active treads; IV, robots based on undulation using vertical waves; $\mathrm{V}$, robots based on undulation using linear expansion.

like locomotion, we can conclude that it has a reasonable cross-section, length, weight and forward velocity. 


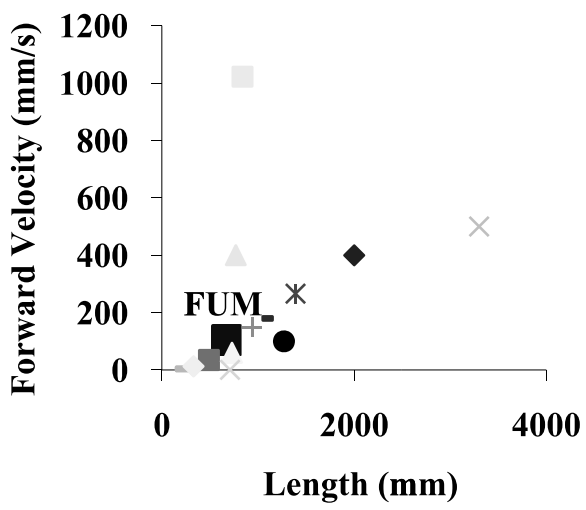

(a)

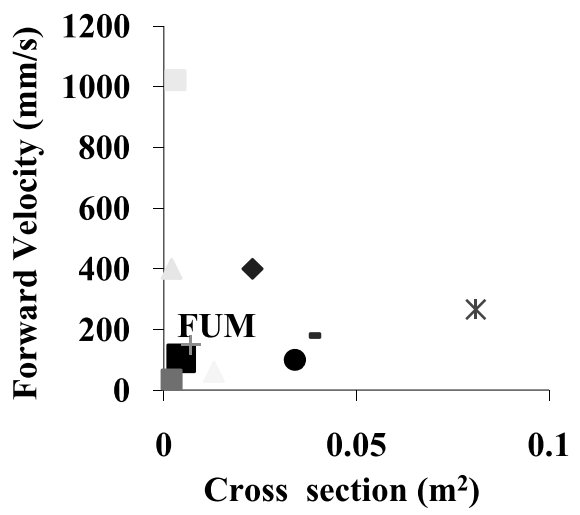

(b)

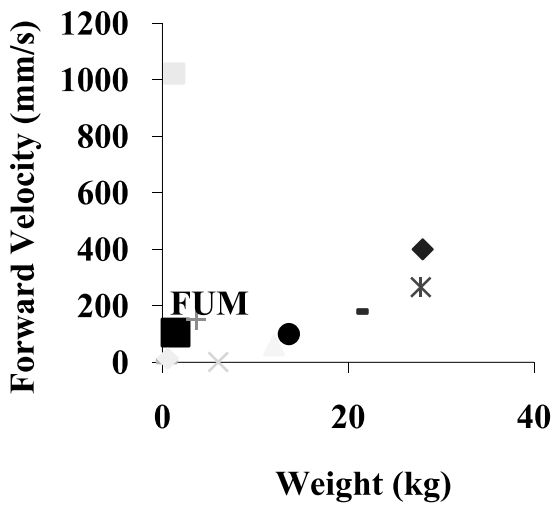

(c)

Figure 13. Maximum velocity versus (a) total length, (b) cross-sectional area and (c) total weight. 口, FUM Snake-3. 


\subsection{Stability}

The stability of snake robots is generally not a big concern. This is because snake robots traveling in the horizontal plane are inherently stable. However, a 2-D snake robot in vertical traveling wave locomotion may not be able to maintain its stability during its full locomotion. In general, when the gravity center of the snake robot falls between two supporting points with the ground, the robot is in a static stable state. It was shown in Ref. [18] that when the number of undulations is equal or greater than 2, there will be at least two supporting points with the ground. If we further add the assumption of equal weight for all robot links, we can then conclude that the center of mass will always be between the two supporting points and, therefore, robot stability is ensured. The stability of FUM Snake-3 is experimentally evaluated. To do this, Displacement of the $X$ - and $Z$-components for the center of mass for undulation numbers $1,1.5$ and 2 are shown in Fig. 14.

As expected, with $K_{n}<2$, the height of the mass center will oscillate as the wave propagates. The motion of the $X$-component of the mass center also shows similar undesirable performance when $K_{n}<2$. It is interesting to note that when $K_{n}$ is equal to 1 versus 2 , the distance travelled is almost cut in half which demonstrates motion deficiency. The effect of $K_{n}$ on the torque of joint 9 is also investigated in Fig. 14c, which shows that when $K_{n}<2$ the motor experiences several high peaks of sudden torque changes that increase as $K_{n}$ decrease. These undesirable impacts are the result of the snake structure physically hitting the ground as it progresses forward. It should be noted that even with $K_{n}=2$ the robot experiences some amount of impact. However, as discussed before, one factor which contributes to this is the shape of the link. Therefore, FUM Snake- 3 is designed with $K_{n}=2$ and curvilinear shaped links. Figure 15 shows snapshots of the simulation and the real snake robot as a function of $K_{n}$.

\section{Conclusions}

In this paper, the kinematics, dynamics, simulation and physical experimentation of worm-like locomotion, also known as a vertical traveling wave, are fully studied. We first present the kinematics of the snake robot. Next, the dynamic model of the snake robot is developed using the Lagrangian method. The derived dynamics formulations are coded in MATLAB software. As joints get closer to the center of gravity, the required maximum torque increases. It was shown that joint 9, the middle link, exhibits the most amount of torque. Therefore, throughout the paper joint 9 is selected and its torque output is reported. Simulation of the snake robot in Webots software is next performed. It is shown that forward vertical traveling wave motion is manifested. Furthermore, the motor torques obtained with Webots and theoretical formulations are compared. Results indicate that the established kinematics and dynamics of the snake robot are reasonable. The details of the FUM Snake-3 robot design are discussed and a novel shaped link is proposed that significantly minimizes the impact forces sensed by the robot. The FUM Snake-3 robot 


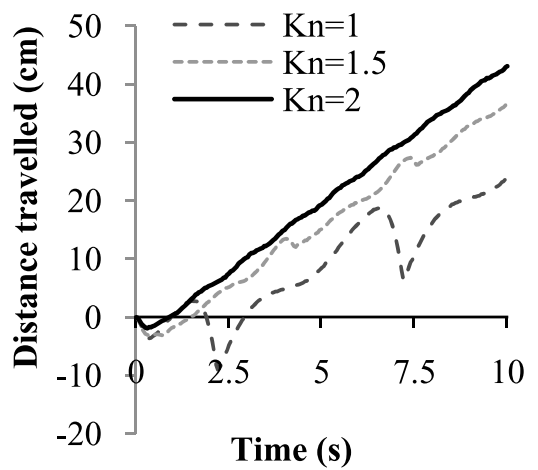

(a)

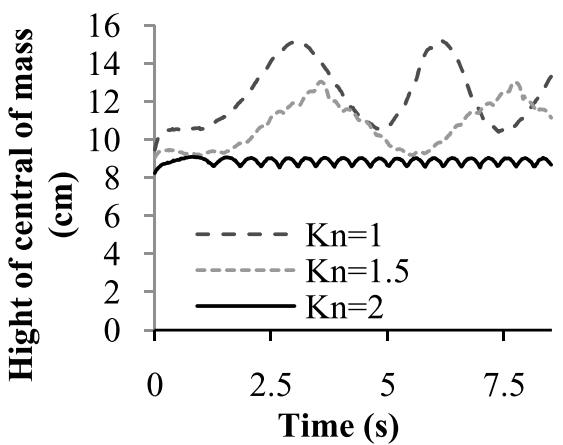

(b)

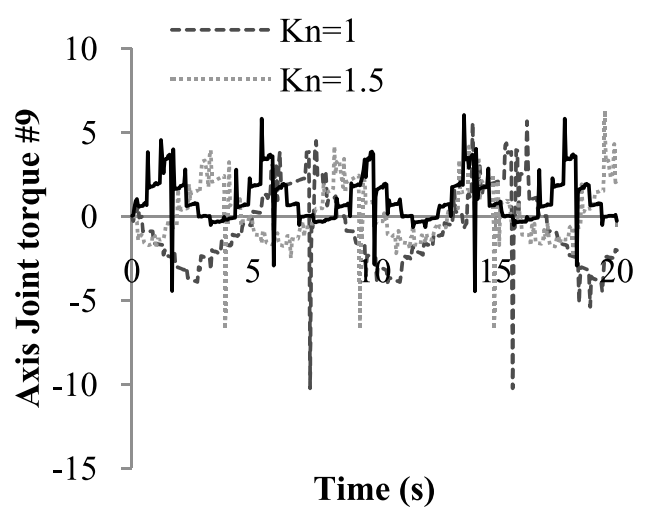

(c)

Figure 14. (a) Mass center $X$-component displacement versus $K_{n}$. (b) Mass center $Z$-component displacement versus $K_{n}$. (c) Torque of joint 9 versus $K_{n}$ using flat links.

is also compared with more well-known snake robots, in particular those having worm-like locomotion. It is shown that FUM Snake- 3 has reasonable geometry and travel speed. Finally, the stability of the FUM Snake-3 robot is experimentally investigated. It was shown that highest level of stability occurs at $K_{n}=2$. 


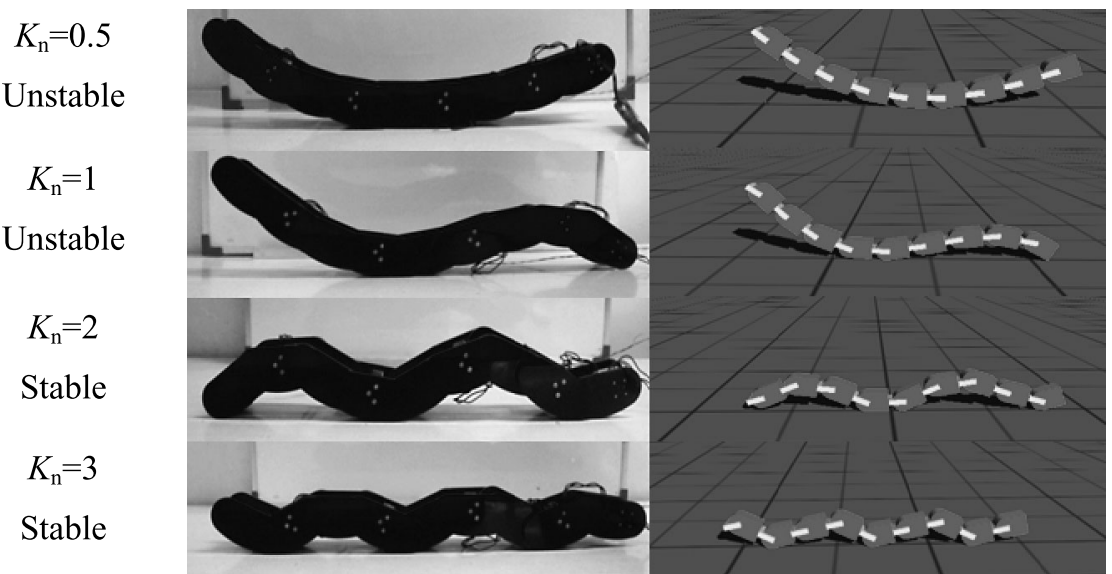

Figure 15. Snapshots of the simulation and the real snake robot as a function of $K_{n}$.

The main contributions of this paper are detailed development of snake kinematics and dynamical equations of worm-like locomotion based on Lagrangian formulations, verification of the dynamic simulation using Webots software, experimental investigation of stability, identifying the effect of link shape, and design details of FUM Snake-3 and its general comparison with other existing snake-like robots.

For our future work, a 3-D version of this robot will be considered. In general, compared with 2-D models, only a few 3-D models have been presented in the published literature $[15,16]$. Clearly the increased number d.o.f. as well as the generalized coordinates of the worm-like robot would increase the complexity of a mathematical model, particularly the dynamics model. Furthermore, the stability analysis of the robot presents additional challenges as the number of contact points is limited.

\section{Acknowledgements}

This work was supported by grant 11619 (19 October, 2008), 'Design and construction of a search and rescue snake robot with optimized locomotion', sponsored by the Ferdowsi University of Mashhad's Research Council.

\section{References}

1. S. Hirose, Biologically Inspired Robots (Snake-Like Locomotor and Manipulator), Oxford University Press, Oxford (1993).

2. L. Chen, S. Ma, Y. Wang, B. Li and D. Duan, Design and modeling of a snake robot in traveling wave locomotion, J. Mech. Mach. Theory 42, 1632-1642 (2007).

3. L. Chen, Y. Wang, S. Ma and B. Li, Analysis of travelling wave locomotion of snake robot, in: Proc. Int. Conf. on Robotics Intelligent Systems and Signal Processing, Changsha, pp. 365-369 (2003). 
4. M. Saito, M. Fukaya and T. Iwasaki, Serpentine locomotion with robotic snakes, IEEE Control Syst. Mag. 22, 64-81 (2002).

5. S. Ma, Y. Ohmameuda and K. Inoue, Dynamic analysis of 3-dimensional snake robots, in: Proc. IEEE/RSJ Int. Conf. on Intelligent Robots and Systems, Sendai, pp. 767-772 (2004).

6. K. J. Dowling, Limbless locomotion: learning to crawl with a snake robot, PhD Dissertation, Carnegie Mellon University (1997).

7. S. Ma, N. Tadokoro, K. Inoue and B. Liz, Influence of inclining angle of a slope to optimal locomotion curves of a snake-like robot, in: Proc. Int. Conf. on Robotics, Intelligent Systems and Signal Processing, Changsha (2003).

8. G. Chirikjian and J. Burdick, The kinematics of hyper-redundant robot locomotion, IEEE Trans. Robotics Automat. 11, 781-793 (1995).

9. G. Chirikjian and J. Burdick, A modal approach to the kinematics of hyper-redundant manipulators, IEEE Trans. Robotics Automat. 10, 343-354 (1994).

10. S. Ma, H. Araya and L. Li, Development of a creeping locomotion of snake-like robot, Int. J. Robotics Automat. 17, 146-153 (2002).

11. S. Hasanzadeh and A. A. Tootoonchi, Ground adaptive and optimized locomotion of snake robot moving with a novel gait, Autonomous Robots 28, 457-470 (2010).

12. H. Kalani, A. Akbarzadeh and J. Safehian, Traveling wave locomotion of snake robot along symmetrical and unsymmetrical body shapes, in: Proc. ISR-Robotik, Munich (2010).

13. J. Safehian, H. Kalani and A. Akbarzadeh, A novel kinematics modeling method for snake robot in traveling wave locomotion, in: Proc. ASME, Turkish (2010).

14. Z. Y. Bayraktaroglu, Snake-like locomotion: experimentations with a biologically inspired wheelless snake robot, Mech. Mach. Theory 44, 591-602 (2009).

15. A. A. Transeth, Modelling and control of snake robots, PhD Dissertation, Trondheim (2007).

16. A. A. Transeth, K. Y. Pettersen and P. Liljebäck, A survey on snake robot modeling and locomotion, Robotica 27, 999-1015 (2009).

17. G. Vossoughi, H. Pendar, Z. Heidari and S. Mohammadi, Assisted passive snake-like robots: conception and dynamic modeling using Gibbs-Appell method, Robotica 26, 267-276 (2008).

18. J. G. Gomez, Modular robotics and locomotion: application to limbless robots, PhD Thesis, Madrid (2008).

19. J. K. Hopkins, B. W. Spranklin and S. K. Gupta, A survey of snake-inspired robot designs, Bionispir. Biomimet. 4, 021101 (2009).

20. B. W. Spranklin, Design, analysis and fabrication of a snake-inspired robot with a rectilinear gait, MS Thesis, University of Maryland (2006).

21. S. Ma, Analysis of creeping locomotion of a snake-like robot, Adv. Robotics 15, 205-224 (2001).

22. S. Ma and N. Tadokoro, Analysis of creeping locomotion of a snake-like robot on a slope, $A u$ tonomous Robots 20, 15-23 (2006).

23. Z. Wang, S. Ma, B. Li and Y. Wang, A unified dynamic model for locomotion and manipulation of a snake-like robot based on differential geometry, Sci. China F 54, 318-333 (2011).

24. P. Liljebäck, Ø. Stavdahl and K. Y. Pettersen, Modular pneumatic snake robot: 3-D modelling, implementation and control, in: Proc. 16th IFAC World Congr., Prague (2005).

25. C. Ye, S. Ma and B. Li, Development of a 3-D snake-like robot perambulator-II: design and basic experiments, in: Proc. IEEE Int. Conf. on Intelligent Mechatronics and Automation, Harbin, pp. 117-122 (2007). 


\section{Appendix}

The detailed final dynamic equation, (40) has a simplified matrix format and can easily be used for any number of links:

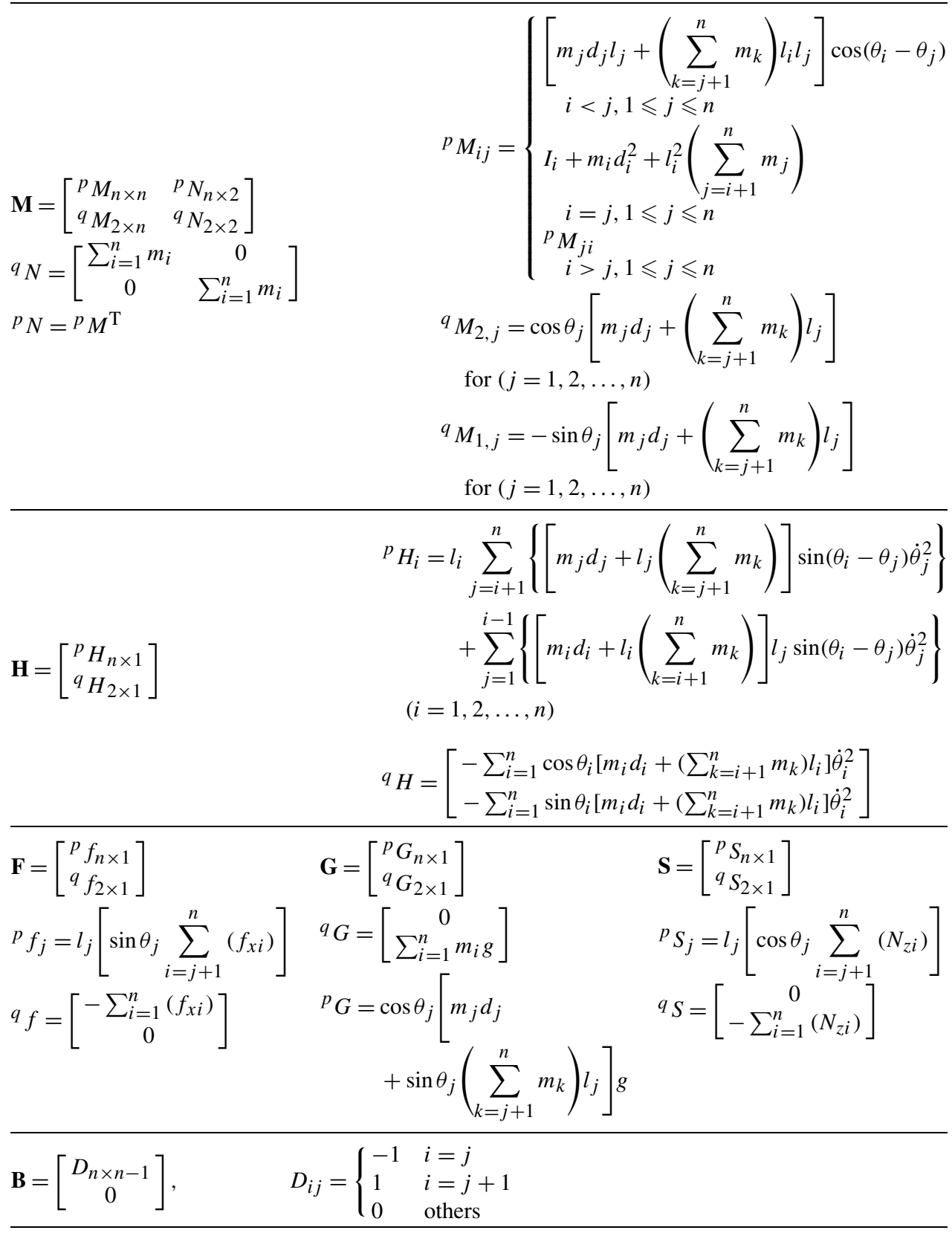




\section{About the Authors}

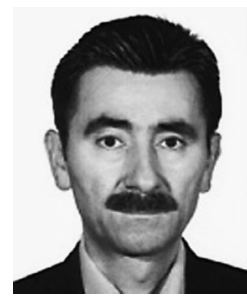

Alireza Akbarzadeh received his $\mathrm{PhD}$ in Mechanical Engineering, in 1997, from the University of New Mexico, USA. He worked at Motorola USA for 15 years where he led R\&D as well as Automation teams. He joined Ferdowsi University of Mashhad, in 2005, and is currently Associate Professor of Mechanical Engineering Department. He has over 18 journal publications and over 50 conference papers. His areas of research include robotics (parallel robots, biologically inspired robots, bipeds), dynamics, kinematics, automation, optimization, design and analysis of experiments.

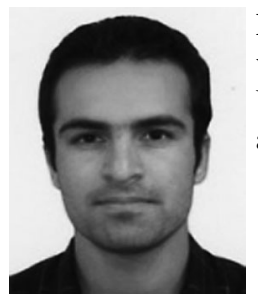

Hadi Kalani received his BS degree in Mechanical Engineering from Azad University of Mashhad, Iran, in 2008. He received his MS degree from Ferdowsi University of Mashhad, Iran, in 2010. His research interest is in the area of robotics and control. 